Volume 7, No.6, November - December 2018

International Journal of Advanced Trends in Computer Science and Engineering

Available Online at http://www.warse.org/IJATCSE/static/pdf/file/ijatcse03762018.pdf

https://doi.org/10.30534/ijatcse/2018/03762018

\title{
Safety and Security for School children's Vehicles using GPS and IoT Technology
}

\author{
${ }^{1}$ N.Saritha Devi, ${ }^{2}$ K.S.R.Raju, ${ }^{3}$ A.Madhu, ${ }^{4}$ R.Raja Sekhar \\ $1,2,3 \& 4$ Department of ECE, Godavari Institute of Engineering \& Technology, Rajahmundry, A.P, India
}

\begin{abstract}
Advanced vehicle monitoring and tracking security system is relied on the android system and embedded board will be intended and executed for observing the vehicle from any area A to area B in real time. The recommended framework works on GPS/GSM $S$ module that incorporates all three things specifically GPS and GSM. The GPS is used to find the present area of the vehicle and it sends the tracing data to the server using GSM technology. The recommended framework might spot inside the vehicle whose location may be should be dictated on the web page and observed at real-time. These frameworks continually see a vehicle's movement and more report cards the status ahead interest. Same time the bulks messages are sent using of the microcontroller, At that point microcontroller issue the control signs will prevent the engine. An obstacle detection system (IR) sensor is generally utilized in mobile applications for calculates the distance. Sensors such as vibration MEMS, alcohol and fire detectors identify the signal in case of an accident occurrence and send a signal to the connected microcontroller vehicle business to keep a trace of the activity of vehicular by sending SMS to the approved mobiles. The recommended framework is also taken care of the passengers' safety with the use of LPG Gas leakage sensor and temperature sensor.
\end{abstract}

Keywords: Controller, Smart Phone, IoT, GPS, GSM

\section{INTRODUCTION}

The Vehicle tracking framework key goal is to provide safety to all vehicles. The accident alert framework primary goal will be saving human in mishaps, this is the better security framework for vehicles. Nowadays, GPS will be extremely useful, this framework empowers the owner to watch and trace his vehicle and identify the movement of vehicle and also track its previous actions [1]. This novel methodology, prominently known as vehicle tracking frameworks that made a number wonders in the vehicle security, which it is not noticeable to anyone who is inside or outside of the vehicle. Therefore it will be utilized as a convert unit that constantly or by any intrude to the framework [2], transfers the area information to the monitoring unit.
Same time those vehicle is theft, those region data starting with following skeleton Might a chance to be used on recognize those zone Furthermore could an opportunity with make educated to police for next action. A portion vehicle following framework Might Significantly distinguish the vehicle's unapproved developments et cetera caution the administrator. This gives an edge through separate pieces for technique to that same reason.

This accident alert system it is used to recognizes the mishap and the position of the mishap occurred and sends GPS to the quantified mobile, workstation and so on. The fire identifier circuit is may be used to recognize the fire in vehicle [3]-[4]; whether the temperature inside the vehicle drives over a particular breaking point then cautioning will be naturally send to the envisioned receiver. The infrared sensor that is also interfaced to the microcontroller will be used to recognize the obstacles and accidents and however whether any incident occurs then its cautioning will be straightforwardly send of the envisioned receiver is shown in figure 1.

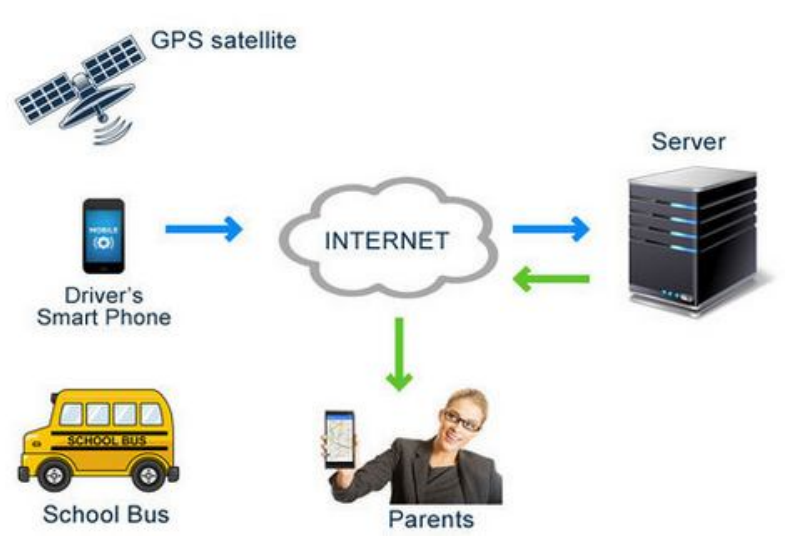

Figure 1: System Block Diagram

This methodology includes communication, GSM, GPS, and others. In any case, we utilize SIM 908 module that is the mixture of 2 units called GSM and GPS [5] The accident alarm framework has 2 parts; the initial part is controlling gadget that sends the communication and the second part is mobile unit that receive the message. The framework 
procedure, data transmission, connections, interfaces, and reception of information among the controlling gadget and mobile unit are working effectively [6].

\section{PROPOSED SYSTEM}

The suggested framework may be utilized for locating and navigating the vehicle with exactness for $10 \mathrm{~m}$. The correct area will be shown in the procedure of latitude and longitude along with the accurate Navigated track on Google map.

\subsection{Vehicle Tracking Features}

It is principally profit for the industries that are relied on transport framework. Since it could display the all vehicle's location in real-time, with the goal that they might make the anticipated information consequently. These tracking framework could store the entire data where the vehicle had gone, where did it stop [7], how much time it take at each stop and might make entirety information analysis. It may be also utilized in trains and buses, to evaluate how much distance are they, and how much time it takes for them to arrive at a specific stop. These frameworks are utilized to information capture, information storage, information analysis and information transfer toward further sensors for example, temperature sensor, gas sensor, liquor sensor and infrared sensors the framework could a chance to be allowed to recognize robbery, fire, and obstacles.

\subsection{Usage of Tracking in India}

Following on India may be essentially used by traffic operators, transport-systems, also taxi dance lover organizations. Taxi drivers specialist drivers use this with assess how inaccessible those vehicle may be from a particular spot also send this information to call-centers, And they may overhaul those normal number something like the separation of the taxi dance specialist position And run through it takes to come to them. Extra use will be to traffic police, in this skeleton is positioned to each vehicle, they might assess those movement through searching on the guide And assuming that whatever incident will be distinguished, at that point they might course the movement under a elective methodology. [8] This tracking is advantageous for the reason that India is one of busy traffic nations and this framework could control numerous number of the traffic issues.

\section{SYSTEM OVERVIEW}

The block diagram of the system vehicle tracking framework represented as in figure 2 . The flow diagram demonstrates the whole perspective of the framework. The blocks that would associate here are GPS, GSM, infrared sensor, Microcontroller, LCD display, control supply, shoot identify

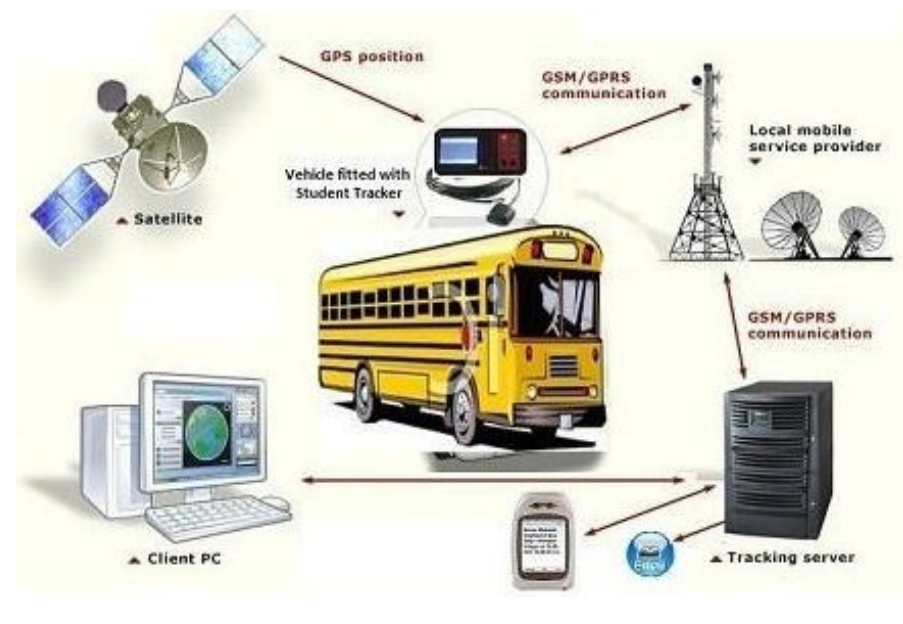

Figure 2: System Working

The main module is GSM and GPS devices are used to receive the message and location information from the satellite in the form of Latitude and longitude real time data [9]. The GSM transfer the SMS to the authorized person. Other module is MEMS sensor it can provides the four directional data to the vehicle location with respect to $\mathrm{x}$-axis, $\mathrm{y}$-axis and $\mathrm{z}$-axis.

Obstacle sensor is used to detect the any obstacle in front of the vehicle to avoid mishaps, alcohol sensor is used to detect range of alcohol if the driver is drunk or not. Temperature sensor is used to monitor the temperature inside the vehicle has caught fire or not

\section{IMPLEMENTATION}

Numerous sorts of gadgets are utilized to design this hardware to make it flawlessly working. Total gadgets are bought from distinctive producers. These parts are soldered on a soldering board. The subsequent lists of hardware are essential for this framework.

\subsection{Temperature Sensor}

The Thermostat is thermally sensitive resistors whose original capacity is to show a massive, predictable and precise modification in electrical resistance when subjected on equivalent progress in human body temperature. The Positive Temperature Coefficient (PTC) thermostats demonstrate expand in electrical resistance when subjected to a rise in the human body temperature and Negative temperature coefficient (NTC) thermostats demonstrate a decrease in electrical resistance when subjected to a rise in human body temperature as in figure 3. 


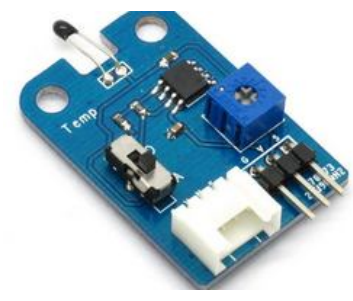

Figure.3.Temperature sensor

\subsection{Alcohol sensor MQ3}

The alcohol sensor MQ3 will be appropriate for identifying alcohol, and this sensor might have a chance to be utilized within a breathalyzer. It has a high sensitivity to alcohol and small sensitivity to benzene. The potentiometer could balance the sensitivity. Sensitive substance of MQ-3 gas sensor as shown in figure 3.1; $\mathrm{SnO} 2$ that with minor conductivity in the fresh air.

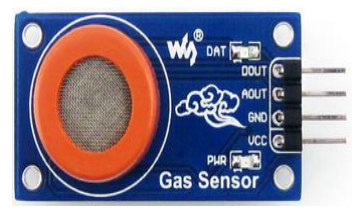

Figure 3.1: Alcohol sensor MQ3

\subsection{Gas sensing module}

The gas sensing module will be used to sense the existence of dangerous gases, for example, CO, LPG, and other hazardous gases inside the vehicle as shown in figure 3.2, If significant levels of gases were found, that is if the $\mathrm{CO}$ exceeds $20 \mathrm{ppm}$ and the level of LPG exceeds 10,000ppm and then the digital information from the gas sensing module may be sent to the MC that shows the data about the gas leakage inside the vehicle and produces an alarm to alert the person inside the vehicle.

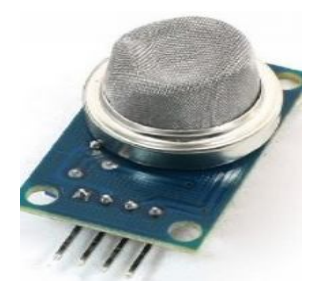

Figure 3.2: Gas sensing module

\subsection{Obstacle Detection}

The obstacle sensing module will be used to sense the static obstacles in front of the vehicle such that, mishaps because of unwanted stopping of the vehicles and collision with trees and different objects particularly during the night time could be avoided. These obstacles might be distinguished by utilizing different strategies for example, such that ultrasonic sensors and so on. [6]. the working standard of the obstacle sensor is shown in Figure 3.3.

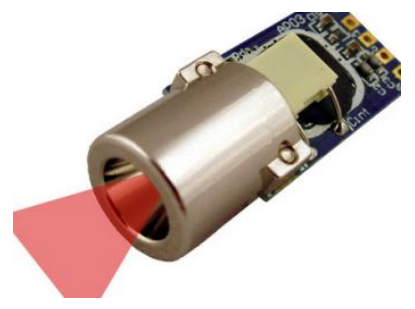

Figure 3.3: IR Sensing Module

An IR module is used to identify the vehicle in front obstacles, it comprises an IR transmitter and a collector that merges to structure an obstacle sensing module.

\subsection{Micro-electro-mechanical sensor (MEMS)}

The Micro-electro-mechanical sensor or MEMS will be a method of mixing electrical and mechanical segments on a chip. As shown in Figure 3.4, MEMS sensor used to identify unexpected vibrations when a mishap happens. It also sends a text message to the official person through the GSM modem associated to the MC such that the official person might take remedy measures and to provide correct medical treatment to them if needed.

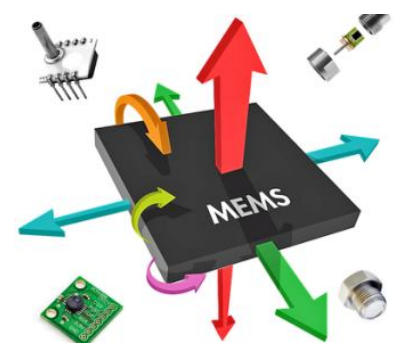

Figure 3.4: Micro electro mechanical sensor

\subsection{GSM Module}

When a GSM modem is linked with a computer, this authorizes the PC to utilize the GSM modem to correspond over the mobile system. Same time these GSM modem is commonly utilized to give mobile web connectivity; many of them might be utilized to sending and getting SMS and MMS messages is shown in Figure 3.5. 


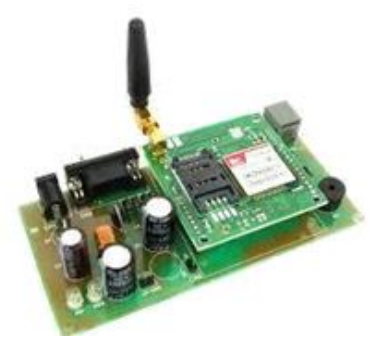

Figure 3.5: GSM Module

\subsection{GPS Module}

As shown in figure 3.6. A GPS navigation gadget is a gadget, which perfectly measures geological area by getting data from GPS satellites. Primarily, it might have been utilized through the United States military, however, now lots of receivers are in the smart phones and automobiles.

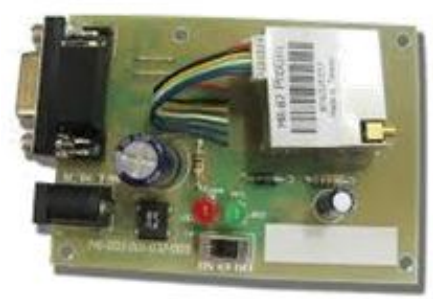

Figure 3.6: GPS MODULE

\subsection{Fire detector}

A fire identifier is a fire alarm gadget designed to react when the transformed thermal energy of fire enhances the heat of the fire sensitive component. The conductivity and thermal mass of the protocol controls the rate flow of temperature into the protocol.

\subsection{Internet of things (IoT)}

The IoT is developing systems of physical objects that have physical connectivity and communication through IP address between webs empowers gadgets and objects [1].

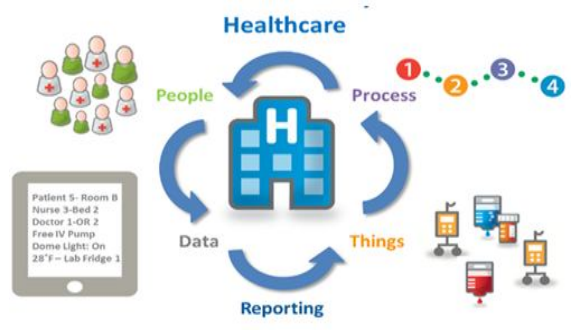

Figure 4: Internet of Things

The suggested framework holds the ESP8266 will be the WiFi module that is utilized to associate to the server. is shown in figure 4, the Wi-Fi system through IoT information will be read on the webpage. To make a simple TCP/IP connection utilizing Hayes-style commands Wi-Fi system will be associated [10] with a little module i.e. MC.

\section{FLOWCHART OF SYSTEM}

The below flow chart can define proposing system. How the systems can be worked shown the below figure 5 .

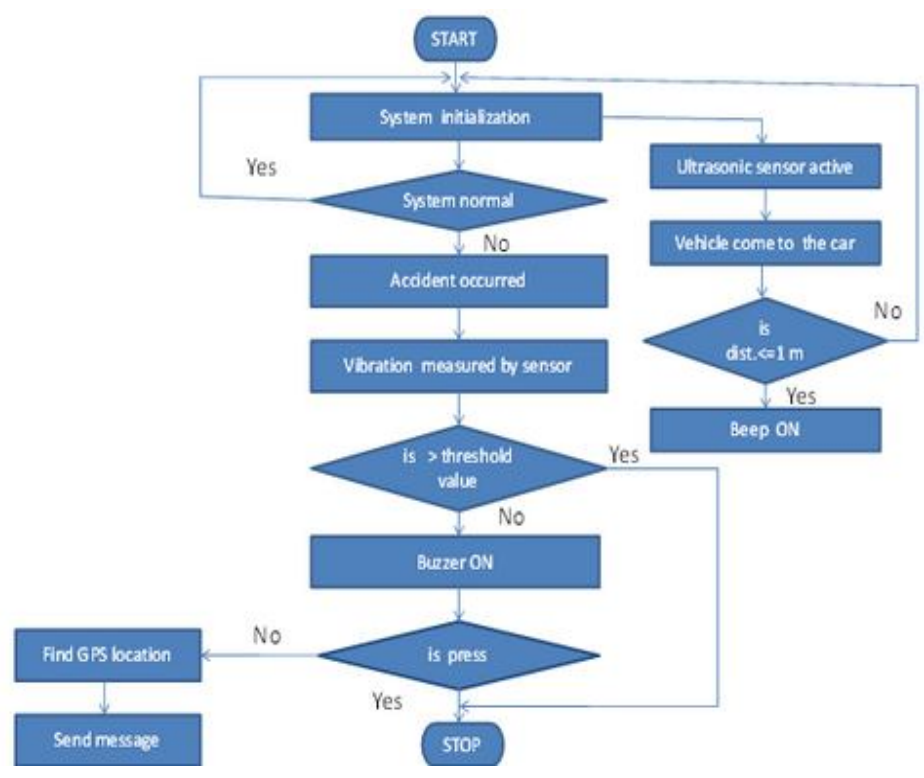

Figure 5: Design Flowchart

\section{RESULTS}

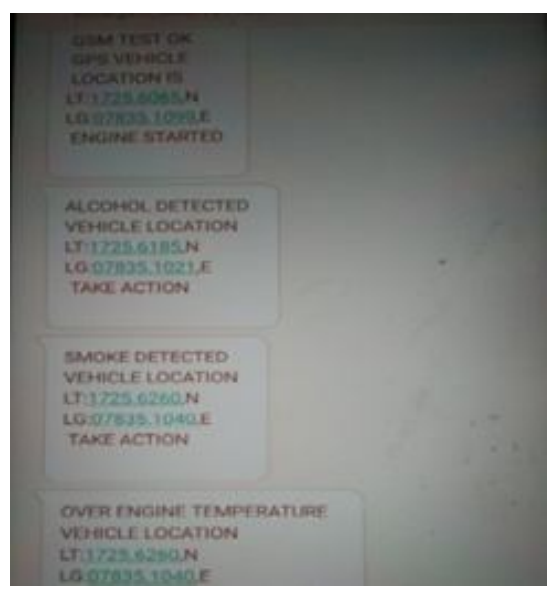

Figure 6: Various sensor information generated using IoT

The ESP8266 will be an ESP8266 with 1 MB regarding inalienable flash, permitting to single-chip unit's proficient of interfacing to $\mathrm{Wi}-\mathrm{Fi}$ as in figure 6. 


\section{CONCLUSION}

A successful result is offered to increase the intellectual framework for vehicles that will display the different parameters of the vehicle in between the consistent timeperiod and will transfer this information of the base unit as clarified in this manuscript, by utilizing hardware platform who's Core is GPS \& GSM module, Android, and alcohol sensor mq3. The designed framework might complete the function of connecting with the base station through GPS, GSM and control of different parameters and also this framework utilized for protection reason of a driver to alert the driver if any fire mischance or any gas leakage, then it inform the driver via buzzer to avoid vehicle from the mishap. The obstacle identification, temperature sensor, and alcohol sensor are utilized to other safety framework in the vehicle. This vehicle tracking and accident caution characteristics play an essential part in daily life.

\section{REFERENCES}

[1] Seokju Lee, Tewolde, G Jaerock Kwon," Design and implementation of vehicle tracking system using GPS/GSM/GPRS technology ad smart phone application", Internet of things (IoT), 2014 IEEE world forum on, Vol. No., pp.353 358, 6-8 March 2014.

[2] R Ramani, S Valarmathy, N SuthanthiraVanitha, S Selvaraju and M Thiruppathi," Vehicle tarcking and locking system based on GSM and GPS", I.J Intelligent systems and Applications, 2013, 09,86-93

[3] A 1 Rashed, M A Omar, O A Singh," A real time GSM/GPS based tracking system based on GSM mobile phone," Future generation communication Technology(FGCT), 2013 second International Conference on, Vol. No., PP. 65,68,12-14 Nov 2013

[4] Tarapiah, S Atalla, S Alsayid,"Smart on board transportation management system Geo- Casting featured", Computer Application and information system
(WCCAIS), 2014 World Congress on, Vol. No.,pp. 1,6, 17-19 Jan 2014

[5] R Kumar, H Kumar, "Availability and handling of data received through GPS device in tracking vehicle", Advance Computing Conference(IACC),2014 IEEE International, Vol., No.,pp.245, 249, 21-22 Feb. 2014

[6] Hoang Dat Pham, M Chi CuongNguyen,'Development of vehicle tracking system using GPS and GSM modem," open systems (ICOS), 2013 IEEE, Vol., no., pp.89, 94, 24 Dec. 2013

[7] Zhigang Shang, Wenli; He, Chao; Zhou, Xiaofeng; Han, Zhonghua; Peng, Hui; Shi, Haibo, "Advanced vehicle monitoring system based on arcgissilverlight," Modelling, Identification \& Control (ICMIC), 2012Proceedings of International Conference on , vol., no., pp.832,836, 24-26 June 2014.

[8] Iman M. Almomani, Nour Y. Alkhalil, Enas M. Ahmad, Rani a M. Jodeh "Ubiquitous GPS Vehicle Tracking and Management System", IEEE Jordan Conference on Applied Electrical Engineering and Computing Technologies (AEECT) 2011

[9] Bhavana Godavarthi, Paparao Nalajala," Wireless Sensors Based Data Acquisition System using Smart Mobile Application," Internet of things, "International Journal of Advanced Trends in Computer Science and Engineering" Vol. 5 No.1, pp. 25-29 Jan 2016

[10] Harshita Chaurasiya, Dr Shivnath Ghosh," Performance Evaluation of Energy-Efficient Cluster based Algorithms in Wireless Sensor Network", International Journal of Advanced Trends in Computer Science and Engineering, Volume 7, No.5, Pp-77-81, 2018.

[11] Papa Rao Nalajala, Rotala Umarani, Naroju Mounika,"Design Of Intelligent Road Traffic Control System For Ambulance Using Rf And Gsm Technology”, International Journal Of Advanced Trends In Computer Science And Engineering, Vol.5 , No.1, Pages : 70-73, (2016) 